\title{
First steps in composite materials for schoolchildren: a STEM educational project
}

Delphine Depuydt ${ }^{\mathrm{a} 1}$, Kevin Hendrickx ${ }^{\mathrm{b}}$, Mahoor Mehdikhani ${ }^{\mathrm{c}}$, Nikolay Petrov ${ }^{\mathrm{c}}$, Stepan V. Lomov ${ }^{\mathrm{c}}$, David Sevenoc

${ }^{a}$ KU Leuven, Department of Materials Engineering, Campus Groep T, Andreas Vesaliusstraat 13, 3000 Leuven, Belgium

${ }^{b}$ KU Leuven, Department of Materials Engineering, Technology Campus De Nayer, Jan De Nayerlaan 5, 2860 Sint-Katelijne-Waver, Belgium

${ }^{c}$ KU Leuven, Department of Materials Engineering, Kasteelpark Arenberg 44, 3001, Leuven, Belgium

\section{Abstract}

Composite materials are inseparable from today's life, yet many schoolchildren (and adults) are not familiar with them. In the framework of InnovationLab, an initiative of KU Leuven, a new ScienceTechnology-Engineering-and-Mathematics (STEM) project was launched, intended to introduce composite materials to schoolchildren. A toolbox was developed which enables teachers to perform composite-related experiments together with their pupils, actualising the pupils' knowledge in chemistry, physics and mathematics. The students learn about polymer matrices and fibres, produce a composite themselves, investigate its mechanical properties and finally test a composite catapult. This way the scientific awareness of the students is raised and they gain insight into today's challenges in composite materials and how engineers respond to them.

\section{Introduction}

Composites nowadays are the material of choice for a wide range of applications, from household appliances to air- and space-crafts. They have high structural capabilities, especially where weight and design freedom matter. Moreover, composites answer society's sustainability challenges because of their lower density and higher durability, causing less energy consumption and less production of greenhouse gases.

Teenagers and young people are not an exception in their exposure to composite materials. They directly benefit from composites, playing with their carbon fibre-reinforced tennis rackets, bathing in glass fibre-reinforced bathtubs, and paddling composite kayaks. They also use composites indirectly, travelling on an aeroplane or a car with composite components and using electricity produced by wind turbines. Despite the considerable level of confrontation with composite materials, a large number of schoolchildren (12-17 years old) do not know about the "composites" concept and its significance to the modern society, economy, science and technology.

\footnotetext{
${ }^{1}$ Corresponding author: Delphine.Depuydt@kuleuven.be
} 
Even though the accessibility to educational science content has drastically increased thanks to the availability of digital media $[1,2]$ and despite considerable efforts dedicated [3] to raise the interest of schoolchildren to Science-Technology-Engineering-and-Mathematics (STEM)-oriented studies, there is still a need to develop activities to increase their interest in studying these fields [8]. To give a more appealing, and realistic, view on STEM, many tools treating eclectic topics have been developed and proposed to non-expert audiences [7-10]. Yet, indicators like PISA [4] highlight a decrease of the average performance of schoolchildren in science and mathematics between 2006 and 2015 in countries like Belgium, France, the Netherlands and the United Kingdom to mention a few. A direct and now well-known consequence is a shortage of engineers capable of managing problem-oriented projects [5, 6]. Indeed, traditional ways of teaching approach STEM as an ensemble of separate subjects (mathematics, physics, chemistry...). Such an approach deviates from the interlaced, interrelated, and interdependent ways the science and technology work.

Composites, by their inherent nature, offer a plethora of possibilities to highlight the interdisciplinary nature of STEM fields and to imprint the interdisciplinary links onto the student's mind. Here we describe an initiative aiming at raising the awareness of the schoolchildren to composite materials through the design, fabrication, characterization, and testing of composite catapult levers.

\section{Composites in STEM educational activities}

Existing STEM educational activities involving composite materials are mostly oriented towards postsecondary students. A number of composite hands-on courses and competitions exist for university students. For instance, in North America, under the umbrella of the SAMPE Bridge Contest, student teams design, build, and test a miniature bridge satisfying a given set of requirements [11]. In World Solar Challenge, student teams from all over the world bring their prototype solar car, which has to drive $3000 \mathrm{~km}$ as fast as possible [12]. STEM programs dealing with composites are available for technical colleges and vocational studies in the USA $[13,14]$; for instance, the National STEM Consortium has "composites" as one of their "high-demand fields" [13]. Some public competitions in aerospace- and ship-modelling [15, 16] are also organized, where composites are a material of choice. However, none of these activities is directly connected with the school curriculum. 
Introduction of composite-related topics in the curriculum of secondary schools is not that common. Understanding of the fundamentals of composite materials asks for a basic knowledge of stress and strain and properties of polymers, which lies beyond the school courses. Placing composites in STEM projects for schools asks for a concise explanation of these concepts, which is challenging. On the brighter side, the environmental impact and sustainability of composites can be put in the focus, which connects with societal problems.

Despite the challenges, some composite-related STEM projects for schoolchildren do exist as a part of or linked to the school curriculum. Here is a non-exhaustive collection of examples. The project "Stemford" in Russia [17] provides online educational modules and "distant experiments" for schoolchildren, including a module (developed by one of the present authors) called "Scales of Composite Materials", which introduces basic mechanical concepts of composites. Composite Technology STEM Program for high schools, from the Composite Prototyping Centre, New York, USA provides schoolchildren with an introduction to the advanced manufacturing of composites [18]. A four-year MINT (German abbreviation for STEM) project in Augsburg schools [19] involves the production of carbon fibre composites, benefiting from being in the "MAI triangle" (Munich - Augsburg - Ingolstadt) of the German composite industry. The Materials World Modules, an integrated interdisciplinary STEM program with National Science Foundation funding in the USA, includes a "Composites" module [20], which takes in total 10 hours of class time in schools.

A STEM project, integrated into the school curriculum, should, on one hand, capitalise on the contents of school mathematics, physics and chemistry, and on the other hand, be appealing for the students. Moreover, the project needs to be applicable in classrooms, considering the limitations in schools. A catapult seems to be a proper choice for a device to build with composites. It is fairly simple, illustrates clearly the performance of materials, and catches the attention of students. Spectacular catapult competitions prove the latter point. A "Punkin Chunkin' Catapult" competition in the USA, with largescale machines and a range up to $1430 \mathrm{~m}$, has been organised for years [21, 22]. In the framework of an educational STEM project for secondary schools in Flanders (Belgium), a catapult, of a much smaller size, which raises no safety concerns, was then chosen as the theme for the composites module and is described here. 


\section{The Catapult Project: an InnovationLab module}

InnovationLab is an initiative of the Faculty of Engineering Science at KU Leuven [23]. Since the start in 2014, it stimulates the schoolchildren's interest in engineering by providing toolboxes enabling secondary schools to run different STEM projects. Unlike other initiatives, the trainers do not go to the school to carry out the workshop with the pupils, but instead, their teachers are trained in the university by trainers that are active in the research field. It is believed that in this way the outreach of the project is larger. Different projects are available, designed in a way to enlighten the students with a social problem and the response of technology on it. Typically 15 toolboxes per project are made, and they can be borrowed free of charge from the university throughout the whole year.

The Composite Materials Group (CMG) of the Department of Materials Engineering put together a toolbox (Figures and tables

Figure 1) on a composite catapult. The project is structured in four sequential modules (Table 1 and Figure 2) and takes half a day to complete. Groups of four pupils each are formed, and they receive a booklet with guidelines and questions.

In Module 1, students explore the properties of different fibres and polymer matrix materials. Connecting one end of a yarn of fibres to a steel bar and the other end to a bucket, the students gradually increase the load on the fibres by adding weights to the bucket. Every weight they add leads to an elongation of the yarn, which is measured with a ruler until the yarn breaks. Plotting the force and the corresponding elongation in a graph, they are introduced to the concepts of stiffness, strain, and strength. The provided fibres in the toolbox are flax and viscose, the former being stiff and strong, the latter being compliant and weak. It was decided not to use glass or carbon fibres, first, as their elongation is low and the precision of a simple ruler measurement is not sufficient, and second, because of the safety issues arising when working with them. Then, the beam-shaped matrix materials, polypropylene and a thermosetting polyurethane, are subjected to simple three-point bending by the same method of adding weights in order to evaluate their stiffness. The main goal of these tests is to let schoolchildren discover different types of polymers and fibres. Later they will compare the composite properties to the polymer materials. Finally, they are asked to select the best combination of materials for their catapult in order to launch a projectile, maximizing the distance travelled by it horizontally. Their decision is based on their gut feeling, where some choose for 
flexible materials and others for stiff materials, which leads to interesting discussions. In the two last modules, they will learn which hypothesis is consistent with physics.

Module 2 envelops the hands-on production. In the first part, the students produce fibre-reinforced thermosetting polyurethane using a low-tech process. They are provided with flax and viscose woven fabrics, which they can incorporate into the matrix. Based on their choice for the optimal fibres from Module 1, each group of students picks either flax or viscose as the reinforcement. When they have calculated (formula provided) the number of fabric layers they need to use, based on the given fibre volume fraction of the composite, a plastic blister mould is used to position the fabric layers properly and the liquid resin is poured over them. Pressure is applied using a top mould and extra weights to reduce the porosity level of the composite. In a second test, the students can experiment with the thermoplastic polypropylene system by melting the polypropylene with a hot iron to impregnate the fibres. Of course, this is only possible for one fibre layer since impregnating multiple layers requires high pressures that cannot be achieved without a mechanical or hydraulic press. In the end, schoolchildren learn how to distinguish between thermoplastics and thermosets and how their different properties determine the production process. All composites the students produce are evaluated in the next modules. The one-layer PP samples are not usable for the testing, therefore the students, after they iron one-layered samples and understand the idea, are given pre-produced polypropylene composite samples with the correct number of layers.

Before the students start firing their projectiles, first the properties of the catapult levers should be determined. This is done in Module 3 by performing simple three-point bending tests on the composites. Conducting the tests enables the students to compare the results to those obtained in Module 1. They will notice a significant increase in stiffness when fibres are incorporated into the polymer. Additionally the density of the composites is determined, by measuring the volume and the weight, and compared to the pure polymer data given in Module 1 . This is the ideal moment for the teacher to explain about light and stiff materials. Then, it is time for the most important part of Module 3, where the actual deformation behaviour of the catapult lever is simulated by a cantilever bending test. The composite beams are fixed at one end in the test set-up provided in the toolbox and loaded at the other end by adding weights. The students measure the deflection of the beam with increasing load. Then, they have to plot the load-deflection curve, from which the energy stored in the material is determined by taking the area underneath the curve. This calculated energy will be used in the last module to theoretically predict the trajectory of the projectile. 
In Module 4, the students predict how far a given projectile travels before touching the ground. To this end, they apply the formulas of constant acceleration and assume that all energy stored in the beam upon deformation is converted into kinetic energy of the projectile. Finally, they fire a light projectile, e.g. a ping-pong or a squash ball, and mark on the ground the distance it travelled. They will find their predictions to be off by approximately a factor of two. The distance travelled is clearly less than what they predicted. Here, they learn that other crucial factors, such as air drag, influencing this distance were neglected in the calculations. By comparing the different materials they have tested so far and the ones provided in the box (glass fibre and carbon fibre composites) they learn that stiff materials store more energy and therefore can shoot a projectile further away. At the end of the day, the teacher summarises the work they have performed and can decide themselves how the pupils will be evaluated.

\section{Evaluation and outcomes}

With the Catapult Project, we succeeded in introducing lightweight composite materials to schoolchildren. This means that pupils became more aware of materials surrounding them and have basic knowledge of advanced materials. With the interactive toolbox, students do not only learn the theory but also experience the material hands-on by making their own catapult.

In the first year (2016-2017), 69 teachers followed the catapult training in KU Leuven, resulting in 46 subsequent toolboxes borrowed for different schools, reaching 834 schoolchildren so far. In Flanders, 460 relevant schools were determined depending on specializations available at the schools. This means that in the first year we were able to reach $10 \%$ of the relevant schools. The estimate for 2017-2018 is to reach the same amount of schools. In the meantime, new projects are being added to the curriculum of InnovationLab, like the newly launched Water Purification Project [23].

The toolbox system is well received by the teachers, as it helps them to meet the fast-changing learning objectives. Besides, the pupils enjoy this alternative teaching method and gain some new skills within the project. Finally, the importance of these STEM projects cannot be neglected as they help to place the current education in a broader context. The following quotes (translated from Dutch) from teachers illustrate the accomplishments of this project.

"These projects have an enormous added value due to the perfect connection to the curricula and the low threshold and easy implementation (thanks to the booklets) for the pupils as well as for the teachers. 
In addition, it fits perfectly with the cross-disciplinary learning objectives." - teacher, Technical Schools Mechelen.

"The involvement of the students during such a project is high. They are driven to show that they can work independently. In this way, they learn both collaborative and problem-solving thinking." - teacher, Guldensporencollege, Kortrijk.

"We find it important to introduce students to lifelike problems through these types of projects and to make them think interdisciplinary." - teacher, Koninklijk Atheneum² Ring, Leuven.

Every year the booklets are evaluated based on the input received while training the teachers and the results of the survey that the teacher receive after performing the project with their pupils. The survey gauges for the age, gender, education direction and enthusiasm of the pupils that performed the project as well as if the school has a STEM education program. Furthermore the clarity of the given information and the level of complexity of the different modules are assessed and feedback and recommendations are asked for. Based on this data, after the first year additional chemistry questions were added to the booklets, responding to a few untapped possibilities to strengthening the link with the chemistry curriculum.

\section{Acknowledgements}

The authors acknowledge the founder of the InnovationLab, Prof. Marian Verhelst and the support from the Faculty of Engineering Science and the Department of Materials Engineering of KU Leuven. The authors would also like to acknowledge the contribution of different members of the Composite Materials Group, which helped to shape this project: Anna Mateeva, Franziska Regel, Steve De Pooter, Katleen Vallons, the technicians Gert Van Beneden and Joop van Deursen which thought of clever solutions to make the setups for the boxes and Manuel Adams who takes care of the maintenance of the boxes. Special thanks to Els Thys for the help and guidance in developing this project to conform to the other toolboxes. Furthermore, we thank the teachers test panel for their input to improve the project and make it fit the curriculum. Lastly, thanks to all the teachers that have and will still participate in the project in order to spread the knowledge of composite materials.

\section{References}

(All websites were accessed in November 2017)

[1] Mar FA, Ordovas-Montanes J, Oksenberg N, Olson AM. The Whiteboard Revolution: Illuminating Science Communication in the Digital Age. Trends Immunol. 2016;37(4):250-3. 
[2] Chintalapati N, Daruri VSK. Examining the use of YouTube as a Learning Resource in higher education: Scale development and validation of TAM model. Telemat Informatics. 2017;34(6):853-60. [3] L. D. Dierking, J. H. Falk. 2020 Vision: Envisioning a new generation of STEM learning research. Cultural Studies of Science Education. 2016;11(1):1-10.

[4] Program for International Student Assessment (PISA). www.oecd.org/pisa/data.

[5] Mellors-Bourne R, May T, Haynes K, Talbot M. Engineering UK: The state of engineering. 2017.

[6] The Education \& Training Foundation. STEM Improvement Programme: Evaluation Report. http://semta.org.uk/images/pdf/STEM-Alliance-Evaluation-Report-Final.pdf.

[7] European Schoolnet. Efforts to Increase Student' Interest in Pursuing Science, Technology, Engineering and Mathematics Studies and Careers: National Measures taken by 30 Countries. 2015.

[8] Scientix: The community for science education in Europe. http://www.scientix.eu/.

[9] National Math + Science Initiative. https://www.nms.org/.

[10] Fondation la main à la pâte: fondation de coopération scientifique pour l'éducation à la science. http://www.fondation-lamap.org/en/international.

[11] SAMPE. SAMPE Student Bridge Contest. http://www.nasampe.org/page/bridgecontest.

[12] Bridgestone Corporation. World Solar Challenge.

https://www.worldsolarchallenge.org/about_wsc/overview.

[13] National STEM consortium. Composites. http://www.nationalstem.org/home/composites/.

[14] IACMI: The Institute for Advanced Composites Manufacturing Innovation. IACMI STEM

Composites Event inspires East TN students. https://iacmi.org/2016/04/22/iacmi-stem-composites-eventinspires-east-tn-students/.

[15] Naviga: World Organisation for Modelshipbuilding and Modelshipsport.

http://www.naviga.org/index.php/en/.

[16] Fédération Aéronautique International: World Air Sports Federation.

https://www.fai.org/sport/aeromodelling.

[17] E-education for nanotechnology industry. Stemford. https://stemford.org/.

[18] Composite Prototyping Center. Composite Prototyping Center Launches Composite Technology Stem High School Technology Program with Vaughn College.

http://compositepro.org/prototyping/composite-prototyping-center-launches-composite-technology-stemhigh-school-technology-program-with-vaughn-college/.

[19] Gymnasium Und Realschule Maria Stern Augsburg. MINT - Mehr Spaß in Naturwissenschaft und Technik. http://www.mariastern.net/Schule/MARIA+STERN/MINT.html.

[20] Materials World Modules. An Inquiry \& Design-Based Science, Technology, Engineering, and Mathematics (STEM) Education Program. https://www.materialsworldmodules.org/index.php/modulesand-user-support/mini-modules/mini-composites-module.

[21] Team ETHOS: Experimental Torsion Hybrid Onager System. http://www.ethoscatapult.com/.

[22] World Championship Punkin Chunkin. https://www.punkinchunkin.com/.

[23] KU Leuven Innovationlab. Innovationlab Ingenieu(r)ze STEM-Projecten voor Secundair Onderwijs https://eng.kuleuven.be/innovationlab.

\section{Figures and tables}

Figure 1 Set of toolboxes for the Catapult Project developed by the Composite Materials Group for InnovationLab.

Figure 2 Setups and working process in the modules: Module 1: tension test (fibres); Module 2: production of the catapult lever; Module 3: measuring properties of the composite catapult levers; Module 4: launching the projectile.

Table 1 Modules of the Catapult project 